\title{
ESTUDO DE FATORES CLÍNICOS PREDITIVOS PARA CRISES EPILÉPTICAS APÓS ACIDENTE VASCULAR CEREBRAL ISQUÊMICO
}

\author{
MARCIA MAIUMI FUKUUIMA*, JOSÉ OSMAR CARDEAL **, \\ JOSÉ GERALDO DE CAMARGO LIMA***
}

\begin{abstract}
RESUMO - Apresentamos aspectos clínicos de 35 pacientes com acidente vascular cerebral isquêmico que evoluiram com crises epilépticas (Grupo 1), comparando-os a 35 pacientes com AVCI sem crises epilépticas (Grupo 2). A comparaç̃̃o das idades entre os grupos não mostrou diferença significante. O sexo masculino e a raça branca predominaram em ambos os grupos. Diabetes melito, hipertensão arterial, ataque isquêmico transitório, acidente vascular cerebral pregresso, enxaqueca, doença de Chagas, embolia cerebral cardiogênica e uso de anticoncepcional oral não diferiram significantemente entre os grupos. Tabagismo e etilismo foram significantemente mais frequentes no Grupo $1(p<0,05)$. O quadro neurológico predominante foi motor, sugerindo comprometimento hemisférico em todos os casos do Grupo 1 e nenhum comprometimento isolado da fossa posterior neste grupo.
\end{abstract}

PALAVRAS CHAVE: acidente vascular cerebral, infarto cerebral, epilepsia, crises epilépticas, etilismo, tabagismo.

\section{Preditive clinical factors for epileptic seizures after ischemic stroke}

ABSTRACT - Preditive clinical factors for epileptic seizures after ischemic stroke. Clinical features of 35 patients with ischemic stroke who developed epilepsy (Group 1) were compared with those of 35 patients with ischemic stroke without epilepsy (Group 2). The age of the patients did not differ between the groups. There were more men than women and more white than other races in both groups. Diabetes melitus, hypertension, transient ischemic attack, previous stroke, migraine, Chagas disease, cerebral embolism of cardiac origin and use of oral contraceptive did not differ between the groups. Smokers and alcohol users were more frequent in Group $1(p<0,05)$. Most patients of Group 1 presented with hemiparesis; none presented cerebellar or brainstem involvement. Perhaps strokes in smokers have some different aspects, that let them more epileptogenic than in non smokers.

KEY WORDS: stroke, cerebral infarction, epilepsy, epileptic seizure, alcoholism, smoking.

Os acidentes vasculares cerebrais (AVC) constituem a terceira causa de morte do mundo e, embora a mortalidade venha diminuindo, estima-se que somente $30 \%$ das pessoas que sofrem AVC recuperem todas as funções afetadas. As principais consequências dos AVC são sequela motora e de linguagem. Porém, podem ocorrer várias outras complicações, entre elas as crises epilépticas. Em 1864 Hughlings Jackson descreveu, pela primeira vez, o aparecimento de crises epilépticas após

Este estudo faz parte da Tese de Mestrado "Crises epilépticas após acidente vascular cerebral por infarto cerebral: estudo de 35 pacientes" 10 , aprovada no curso de pós-graduaçåo da Neurologia da Escola Paulista de Medicina (EPM) da Universidade Federal de São Paulo (UNIFESP): *Mestre em Neurologia pela EPM-UNIFESP; **Professor Adjunto da Disciplina de Neurologia da EPM-UNIFESP; ***Professor Titular da Disciplina de Neurologia da EPM-UNIFESP. Estudo realizado no Setor de Doenças Neurovasculares da Disciplina de Neurologia da EPM-UNIFESP. Aceite: 22-fevereiro-1996. 
instalação de hemiplegia provocada por embolia cerebral na artéria cerebral média e, em 1885, foi publicada por William Gowers a primeira série de pacientes com hemiplegia e epilepsia ${ }^{14}$. Os AVC constituem uma das causas mais importantes de epilepsia de início tardio, sendo observados em $14 \%$ dos epilépticos com idade superior a $25 \operatorname{anos}^{6}$ e em cerca de $30 \%$ dos epiléticos com mais que $60 \operatorname{anos}^{11.15 .22}$. A análise da literatura sobre o tema é dificultada pelo uso pouco adequado de termos como epilepsia vascular, epilepsia precursora, epilepsia irritativa, entre outros, e da inclusão de vários tipos de $\mathrm{AVC}$, indiscriminadamente, já que os mecanismos fisiopatológicos podem ser distintos.

Este estudo tem o objetivo de avaliar aspectos clínicos, tais como antecedentes pessoais com ênfase a fatores de risco para AVC, pressão arterial e tipo de manifestação neurológica, de pacientes com acidente vascular cerebral isquêmico (AVCI) que evoluíram com crises epilépticas, comparandoos com os de pacientes com AVCI sem crises epilépticas, com o intuito de detectar fatores preditivos para a ocorrência de crises epilépticas.

\section{CASUISTICA E MÉTODOS}

Foram estudados 35 pacientes atendidos no período de 1990 a 1992 que apresentaram crises epilépticas com início após 24 horas da instalação do AVCI e com pelo menos uma recorrência da crise (Grupo 1, G1). Estes foram comparados a 35 pacientes controle (Grupo 2, G2), que não apresentaram crises após o AVCI por periodo de até 1860 dias, já que o critério para definir o grupo controle foi o tempo entre o AVCI e a primeira crise epiléptica. Optamos por estudar as crises de ocorrência após 24 horas da instalação do AVCI porque as crises que ocorrem nesse período inicial costumam ser decorrentes de fatores outros que não o AVCI (distúrbios metabólicos, hemodinâmicos, abstinência a drogas e álcool, por exemplo).

Todos os diagnósticos de AVCI foram feitos pelo quadro clínico e tomográfico e classificados segundo a Classificação de Doenças Cerebrovasculares III, de 1990, do National Institute of Neurological Disorders and Stroke ${ }^{26}$. Foram excluidos pacientes que desenvolveram sindrome de abstinência, ou submetidos a intervenções cirúrgicas intracranianas ou a cirurgias neurovasculares, ou com história de crises epilépticas prévias ao AVCI, ou que proporcionaram informaçðes insuficientes para o diagnóstico de crises epilépticas, ou com antecedente de encefalopatia por trauma cranioencefálico, anóxia, infeç̧ôes e tumor, ou portadores de neurocisticercose, ou cujo líquido cefalorraquidiano ou tomografia computadorizada de crânio foram sugestivos de lesåo hemorrágica, inflamatória, tumoral ou traumática, e que tenham tido crises epilépticas apenas durante a instalação do AVC.

Todos os pacientes foram submetidos a anamnese e exame clínico e neurológico. Nos antecedentes mórbidos pessoais, foi dado especial enfoque aos fatores de risco para doenças vasculares, tais como hipertensão arterial, diabetes melito, ataque isquêmico transitório (AIT), AVC pregresso, enxaqueca, uso de anticoncepcional oral, doença de Chagas comprovada sorologicamente, cardiopatia como possível causa de embolia cerebral, etilismo (que foi considerado positivo quando o paciente referiu uso de pelo menos uma dose de bebida alcoólica diariamente) e tabagismo (quando o paciente referiu uso de qualquer quantidade de cigarros diariamente).

Para análise dos resultados foram aplicados os seguintes testes: 1 . Teste de Mann-Whitney ${ }^{23}$, com a finalidade de comparar duas a duas, as variáveis estudadas; este teste foi feito com aproximação à curva normal (z) quando o tamanho da amostra assim o exigiu. 2. Teste do quiquadrado para tabelas $2 \times 2$, com a finalidade de analisar as associaçðes entre as variáveis estudadas; quando observadas as restrições de Cochran, foi aplicado 0 teste exato de Fisher $^{23}$. 3. Teste do quiquadrado para tabelas de contingência ${ }^{23}$, com a finalidade de comparar os dois grupos estudados em relação ao quadro neurológico. O nível de significância foi fixado em 0,05 ou $5 \%(\alpha<0,05)$.

\section{RESULTADOS}

O sexo masculino predominou tanto no $\mathrm{G} 1(57,1 \%)$ como no $\mathrm{G} 2(68,6 \%)$, porém sem diferença estatisticamente significante entre os grupos. A idade variou de 32 a 85 anos no $\mathrm{Gl}$ e de 20 a 84 anos no G2, e foram comparadas segundo o sexo (Tabela 1). Houve predomínio da raça branca em ambos os grupos $(87,7 \%$ no G1 e $74,3 \%$ no G2).

$A$ análise dos antecedentes pessoais é mostrada na Tabela 2.

Os valores das medidas de pressão arterial sistólica não mostraram diferença significante entre os grupos, porém os dados sugerem que entre os homens, seja maior no G2 do que no G1 ( $Z$ 
Tabela l. Número de pacientes $(N)$, média $(X)$ e mediana $(M i)$ das idades, e resullado do teste de MannWhitney para idades segundo o sexo.

\begin{tabular}{clll}
\hline & Grupo 1 & Grupo 2 & G1 X G2 \\
\hline \multirow{3}{*}{ Masculino } & $N=20$ & $N=24$ & \\
& $X=54,6$ & $X=60,1$ & Z calculado $=1,85$ (NS) \\
& $M i=57,0$ & $M i=63,0$ & \\
Feminino & $N=15$ & $N=11$ & \\
& $X=60,6$ & $X=51,9$ & U calculado $=47,5$ (NS) \\
Mi $=63,0$ & Mi $=49,0$ & \\
& Z calculado $=1,36(\mathrm{NS})$ & $\mathrm{Z}$ calculado $=2,15(\mathrm{M}>\mathrm{F})$
\end{tabular}

NS, não significante; $M$, masculino; F, feminino; G1, grupo 1; G2, grupo2

Tabela 2. Resultado do teste exato de Fisher $e$ do teste do qui-quadrado $\left(x^{2}\right.$ crítico $=3,84$ ) para fatores de risco para crises epilépticas após AVC.

\begin{tabular}{|c|c|c|c|c|}
\hline & \multicolumn{2}{|c|}{$\mathrm{MXF}$} & \multicolumn{2}{|c|}{$\mathrm{G} 1 \times \mathrm{X} 2$} \\
\hline & G1 & G2 & M & $\mathrm{F}$ \\
\hline Diabetes melito & NS & NS & NS & NS \\
\hline Ataque isquêmico transitório & NS & NS & NS & NS \\
\hline AVC pregresso & NS & NS & NS & NS \\
\hline Hipertensão Arteiral & NS & NS & NS & NS \\
\hline Enxaqueca & NS & NS & NS & NS \\
\hline Doença de Chagas & NS & NS & NS & NS \\
\hline Embolia & NS & NS & NS & NS \\
\hline Anticoncepcional & NS & NS & NS & NS \\
\hline Tabagismo & $\mathrm{p}<0,01(\mathrm{M}>\mathrm{F})$ & NS & $\mathrm{p}<0,02(\mathrm{G} 1>\mathrm{G} 2)$ & NS \\
\hline Etilismo & $\mathrm{p}<0,01(\mathrm{M}>\mathrm{F})$ & NS & $p<0,02(G 1>G 2)$ & NS \\
\hline
\end{tabular}

NS, não significante; $M$, masculino; $F$, feminino; $G 1$, grupol; $G 2$, grupo2

Tabela 3. Quadro neurológico predominante: resultado do teste do qui-quadrado.

\begin{tabular}{|c|c|c|c|c|c|c|}
\hline & \multicolumn{2}{|c|}{ Grupo 1} & \multicolumn{2}{|c|}{ Grupo 2} & \multicolumn{2}{|c|}{ Total } \\
\hline & $N$ & $\%$ & $\mathbf{N}$ & $\%$ & $\mathrm{~N}$ & $\%$ \\
\hline Motor Esq & 13 & 38,2 & 9 & 25,7 & 22 & 31,9 \\
\hline Motor Dir + Afasia & 22 & 61,8 & 22 & 62,9 & 44 & 62,3 \\
\hline Ataxia & 0 & 0,0 & 4 & 11,4 & 4 & 5,8 \\
\hline Total & 35 & 100 & 35 & 100 & 70 & 100 \\
\hline
\end{tabular}

Esq, esquerdo; Dir, direito; $X^{2}$ calculado $=4,74 ; X^{2}$ critico $=5,99$ 
calculado $=1,80 ; Z$ crítico $=1,96)$. A pressão arterial diastólica dos homens foi maior nos pacientes do $\mathrm{G} 2$ que nos do $\mathrm{G} 1$ ( $\mathrm{Z}$ calculado=2,17; $\mathrm{Z}$ crítico=1,96).

A Tabela 3 mostra os resultados da avaliação neurológica. Ressalta o fato de que nenhum paciente do Gl tenha apresentado quadro neurológico sugestivo de comprometimento da fossa posterior.

\section{DISCUSSÃO}

Os dados obtidos na casuística apresentada parecem seguir a tendência epidemiológica para AVC no mundo, isto é, ocorrência em indivíduos idosos, com ligeiro predomínio no sexo masculino. Os AVC constituem a etiologia mais frequente de crises epilépticas em pacientes idosos, variando de 17 a $52 \%$, seguidos de tumor ( 0 a $29 \%$ ), causa desconhecida ( 11 a $65 \%$ ), trauma ( 3 a $18 \%$ ) e causa tóxica ou metabólica ( 2 a $18 \%)^{5,9}$. Outros fatores como etilismo, atrofia cerebral, infecções, demência e hematoma subdural constituem somente 1 a $2 \%$ das etiologias de epilepsia no idoso ${ }^{21}$.

$\mathrm{Na}$ amostra estudada, o antecedente de diabetes melito apareceu em igual número nos dois grupos e o de hipertensão arterial, em número bastante próximo; também não houve diferença entre o grupo epiléptico e o grupo controle, para a pesquisa de tromboembolismo cerebral de origem cardíaca e mesmo especificamente para a doença de Chagas, uma das mais frequentes cardiopatias no Brasil, causadora de cardiomiopatia dilatada, por vezes com aneurismas de parede ventricular e arritmia, entre outras complicações. Embora alguns autores enfatizem a importância da embolia cerebral de origem cardíaca para o aparecimento de crises epilépticas após o $\mathrm{AVC}^{12,16,18}$, o diagnóstico diferencial entre embolia cerebral e trombose de vasos cerebrais é difícil e nem sempre possível ${ }^{17,19}$.

Há poucos estudos a respeito de aspectos clínicos especificamente para epilepsia após AVCI. Foram relatados alguns antecedentes que predispõem à ocorrência de epilepsia, principalmente os relacionados à embolia cerebral de origem cardíaca. Uma das teorias para explicar tal fato diz que a embolia cerebral de origem cardíaca frequentemente resulta em infarto hemorrágico, e este comportase como hemorragia intraparenquimatosa ou subaracnoídea, elevando o risco de epilepsia ${ }^{2,3,7}$. Cocito et al. concluíram que cofatores de morbidade para AVCI pesquisados, ou seja, diabetes melito, hipertensão arterial, aterosclerose e arritmia cardíaca não se relacionam à presença de crises epilépticas durante a evolução do AVC, mesmo incluindo as duas primeiras semanas desde a instalação ${ }^{4}$.

No presente estudo foi nítida a associação entre pacientes com AVCI que evoluíram com epilepsia e etilismo e tabagismo. O etilismo pode associar-se à ocorrência de crises epilépticas por alguns mecanismos descritos, como atrofia cortical ${ }^{1,21,24}$, abstinência alcoólica ${ }^{25}$, ou complicações como lesões traumáticas, infecciosas e vasculares ${ }^{13}$. Cicatrizes corticais são comuns em etilistas, decorrentes de pequenos traumas. Se essas cicatrizes não forem suficientemente epileptogênicas por si só, podem também causar crises parciais durante a abstinência alcoólica ${ }^{8}$. Sabe-se que o tabagismo é um potente fator de risco para a ocorrência de cardiopatias, e estas últimas para embolias cerebrais. Assim, poderia ser especulado que as crises epilépticas por AVCI em tabagistas poderiam estar relacionadas a fenômenos embólicos, porém não está comprovado que as embolias cerebrais de origem cardíaca produzam mais epilepsia do que as tromboses de vasos cerebrais. Os nossos resultados sugerem que quando o AVCI ocorre em tabagistas, estes se tornam suscetíveis a crises epilépticas, o que indica que o AVCI nesses individuos pode apresentar características diferentes das que ocorrem em não fumantes. É interessante que a influência do tabagismo nas epilepsias não tenha sido estudada, possivelmente porque a maioria das epilepsias se inicia antes da aquisição do tabagismo pela maioria dos indivíduos.

$\mathrm{O}$ antecedente de AIT prévio ao infarto cerebral em questão, esteve presente em $31 \%$ dos pacientes do Grupo 1 e em 17\% no Grupo 2, e o antecedente de AVC pregresso, em 43\% no Grupo 1 e em 23\% no Grupo 2. Possivelmente, devido ao número de casos estudados, estes dados não tiveram significância estatística, apesar da presença de doenças cerebrovasculares pregressas no grupo de pacientes com epilepsia parecer ter importância. 
No nosso estudo, o quadro clínico predominante foi motor, sem diferença significante entre o dimídio comprometido. Chama-nos a atenção a ocorrência de déficits neurológicos relacionados ao território carotídeo em $100 \%$ dos casos de pacientes do Grupo 1 e a ausência de sinais sugestivos de comprometimento vertebrobasilar nesses pacientes. Este dado é concordante com a literatura, que relaciona epilepsia após AVC a lesões do território carotídeo e não do território vertebrobasilar, tanto em estudos clínicos como anatomopatológicos ${ }^{4,19}$.

Agradecimento - Agradecemos ao Prof. Dr. Neil Ferreira Novo à e Profa. Dra. Yara Juliano pela orientação da análise estatística.

\section{REFERÊNCIAS}

1. Advaloff $W$. Cerebral atrophy and alcohol-withdrawal epilepsy: a clinical and computer tomography study. In Meinsardi H, Rowan A (eds). Advances in epileptology. Amsterdam: Swets and Zeitlinger, 1978:389-393.

2. Cerebral Embolism Study Group. Immediate anticoagulation of embolic stroke: a randomized trial. Stroke 1983;14:668-676.

3. Cerebral Embolism Study Group. Immediate anticoagulation of embolic stroke: brain hemorrhage and management options. Stroke 1984;15:779-789.

4. Cocito L, Favale E, Reni L. Epileptic seizures in cerebral arterial occlusive disease. Stroke 1982;13:189-195.

5. Courjon J, Artru F, Zeskov P. A propos des crises d'epilepsie apparaissant aprés 60 ans observées en clientèle de neurologie dans un service de neurochirurgie. Sem Hop Paris 1970;46:3129-3132.

6. Dams AM, Fuglsang-Frederickson A, Svarre-Olsen U, Dam M. Late onset epilepsy, etiologies, types of seizure and value of clinical investigation, EEG and CT scan. Epilepsia 1986;26:227-231.

7. Davis KR, Taveras JM, New PFJ. Cerebral infarction diagnosis by computerized tomography. Am J Roentgenol 1975;124:643-660.

8. Engel J. Seizures and epilepsy. Philadelphia: Davis, 1989:112-134.

9. Fuerstein J, Weber M, Kurtz D, Rohmer F. Etude statistique des crises épileptiques apparaisant apres l'âge de 60 ans. Sem Hôp Paris 1970;46:3125-3128.

10. Fukujima MM. Crises epilépticas após acidente vascular cerebral por infarto cerebral: estudo de 35 pacientes. Tese de Mestrado, Escola Paulista de Medicina, Universidade Federal de São Paulo. São Paulo, 1994.

11. Hauser WA, Kurland LT. The epidemiology of epilepsy in Rochester, Minnesota, 1953 through 1967. Epilepsia 1975;16:1-66.

12. Holmes GL. The electroencephalogram as a predictor of seizures following cerebral infarctions. Clin Electroencephalogr 1980;11:83-86.

13. Janz D, Masuhr KF. Epilepsia tarda: a clinical, EEG and neuro-radiological study (taking in consideration cranial computerized tomography). Epilepsia 1978;19:110.

14. Lesser R, Luders H, Dinner DS, Morris HH. Epileptic seizures due to thrombotic and embolic cerebrovascular disease in older patients. Epilepsia 1985;26:622-630.

15. Luhdorf F, Jensen LK, Plesner A. Etiology of seizures in the elderly. Epilepsia 1986;27:458-463.

16. Meyer JS, Charney JZ, Riviera VM, Mathew NT. Cerebral embolization: prospective clinical analysis of 42 cases. Stroke 1971;2:541-554.

17. Mohr JP, Caplan LR, Melski JW, Goldstein RJ, Duncan GW, Kistler JP, Pessin MS, Bleich HL. The Harvard cooperative stroke registry: a prospective registry. Neurology 1978;28:754-762.

18. Olsen TS, Hogenhaven H, Thage O. Epilepsy after stroke. Neurology 1987;37:1209-1211.

19. Richardson EP, Dodge PR. Epilepsy in cerebral vascular disease: a study of the incidence and nature of 104 consecutive proven cases of cerebral infarction and hemorrhage. Epilepsia 1954;3:49-74,.

20. Ron MA, Acker W, Shaw GK, Lishman WA. Computerized tomography of the brain in chronic alcoholism: a survey and follow up study. Brain 1982;105:497-514.

21. Scheuer ML, Cohen J. Seizures and epilepsy in the elderly. Neurol Clin 1993;11:787-804.

22. Shinton RA, Gill JS, Zezulka AV, Beevers DG. The frequency of epilepsy preceding stroke: case control study in 230 patients. Lancet 1987;1:11-12.

23. Siegel S. Estadistica no parametrica. México: Trillas, 1975.

24. Torvick A, Klindboe CF, Rodge S. Brain lesions in alcoholics: a neuropathological study with clinical correlation. J Neurol Sci 1982;56:233-248.

25. Victor $M$, Brausch $C$. The hole of abstinence in the genesis of alcoholic epilepsy. Epilepsia 1967;8:1-20.

26. Whisnant JP, Basford JR, Bernstein EF, Cooper ES, Dyken ML, Easton JD, Little JR, Marler JR, Millikan CH, Petito CK, Price TR, Raichle ME, Robertson JT, Thiele B, Walker MD, Zimmerman RA. Special report from the National Institute of Neurological Disorders and Stroke: Classification of Cerebrovascular Disease III. Stroke 1990;21:637-676. 